\title{
Alpha Inelastic Scattering and Cluster Structures in Light Nuclei
}

\author{
T. Kawabata \\ Department of Physics, Kyoto University, Kyoto 606-8502, Japan
}

\begin{abstract}
The cluster structures of the excited states in ${ }^{11} \mathrm{~B}$ and ${ }^{13} \mathrm{C}$ were discussed by measuring the isoscalar monopole strengths in the inelastic $\alpha$ scattering at $E_{\alpha}=388 \mathrm{MeV}$. It was found that the $1 / 2_{2}^{-}, 1 / 2_{3}^{-}$, and $1 / 2_{4}^{-}$states in ${ }^{13} \mathrm{C}$ are candidates for the $\alpha$ cluster states with a $3 \alpha+n$ molecular configuration.
\end{abstract}

\section{Introduction}

Alpha particle clustering is an important concept in nuclear physics for light nuclei. On the basis of the Ikeda diagram [1], the $\alpha$ cluster structure is expected to emerge near the $\alpha$-decay threshold energy in self-conjugate $A=4 n$ nuclei. For example, it has been suggested that the $7.65-\mathrm{MeV} 0_{2}^{+}$state in ${ }^{12} \mathrm{C}$, which locates at an excitation energy higher than the $3 \alpha$-decay threshold by $0.39 \mathrm{MeV}$, has a $3 \alpha$-cluster configuration [2]. Recently, the cluster models have been applied to the neutron-rich nuclei, and the molecular structures where the excess neutrons act as the covalent particles have been discussed.

Milin and von Oertzen proposed $\alpha$ cluster states in ${ }^{13} \mathrm{C}$ with one covalent neutron on the basis of the compiled experimental data, and proposed the $K=3 / 2^{-}$and $K=3 / 2^{+}$molecular bands [3]. They also pointed out that the $1 / 2_{2}^{-}$state at $E_{x}=8.86 \mathrm{MeV}$ and the $1 / 2_{2}^{+}$state at $E_{x}=10.996 \mathrm{MeV}$ in ${ }^{13} \mathrm{C}$ are considered to be the $3 \alpha+n$ cluster states where an excess neutron behaves as a covalent particle in the $1 p_{1 / 2}$ and $2 s_{1 / 2}$ orbits around the $0_{2}^{+}$state in ${ }^{12} \mathrm{C}$. They suggested that the $1 / 2_{2}^{-}$and $1 / 2_{2}^{+}$states in ${ }^{13} \mathrm{C}$ may have the triangular shape since the covalent neutron plays a role to stabilize the three $\alpha$-particle structure to a triangular shape in these states. Thus, a comparative study between the $0_{2}^{+}$state in ${ }^{12} \mathrm{C}$ and the two states in ${ }^{13} \mathrm{C}$ is important to examine the molecular structure in the atomic nuclei.

On the other hand, although the molecular states built on the $0_{2}^{+}$states were proposed, no candidate for the molecular state on the $0_{3}^{+}$states at $E_{x}=10.3 \mathrm{MeV}$ in ${ }^{12} \mathrm{C}$ has been observed in ${ }^{13} \mathrm{C}$. It is also important to search for those states from a view of the cluster model.

A cluster state relevant to the $0_{2}^{+}$state in ${ }^{12} \mathrm{C}$ was suggested in ${ }^{11} \mathrm{~B}$ as well as ${ }^{13} \mathrm{C}$ [4]. The $3 / 2_{3}^{-}$ state at $E_{x}=8.56 \mathrm{MeV}$, which is not predicted by the shell-model calculation by Cohen and Kurath [5] is predominately excited by the $\Delta J^{\pi}=0^{+}$transition in the ${ }^{11} \mathrm{~B}\left(d, d^{\prime}\right)$ reaction [6]. The angular distribution of the $\left(d, d^{\prime}\right)$ cross section for the $3 / 2_{3}^{-}$state in ${ }^{11} \mathrm{~B}$ is very similar to that for the $0_{2}^{+}$state in ${ }^{12} \mathrm{C}$. This fact indicates the $3 / 2_{3}^{-}$state is considered to be an $\alpha$ cluster state with a proton hole in the $1 p_{3 / 2}$ orbit coupled to the $0_{2}^{+}$state in ${ }^{12} \mathrm{C}$, while the ground state in ${ }^{11} \mathrm{~B}$ is considered to have a proton hole in the $1 p_{3 / 2}$ orbit coupled to the ground state in ${ }^{12} \mathrm{C}$.

For clarification of the cluster structure in ${ }^{13} \mathrm{C}$ and ${ }^{11} \mathrm{~B}$, further information on the natural-parity excitation strengths is indispensable. Especially, the isoscalar monopole strength is a key ingredient because it is expected that the $\alpha$ cluster states are excited from the ground state by the monopole transitions $[4,7]$.

In the present work, the isoscalar monopole strengths in ${ }^{13} \mathrm{C}$ and ${ }^{11} \mathrm{~B}$ were obtained by measuring the inelastic $\alpha$ scattering at $E_{\alpha}=388 \mathrm{MeV}$, and the $\alpha$ cluster structure in ${ }^{13} \mathrm{C}$ and ${ }^{11} \mathrm{~B}$ was discussed.

\section{Experiment}

The experiment was performed at the Research Center for Nuclear Physics, Osaka University, using a 388-MeV $\alpha$ beam. The $\alpha$ beam extracted from the ring cyclotron was achromatically transported to selfsupporting ${ }^{11} \mathrm{~B}$ and ${ }^{13} \mathrm{C}$ targets with the thicknesses of $16.7 \mathrm{mg} / \mathrm{cm}^{2}$ and $1.5 \mathrm{mg} / \mathrm{cm}^{2}$. Scattered $\alpha$ particles were momentum analyzed by the high-resolution spectrometer Grand Raiden [8]. The focal-plane 
detector system of Grand Raiden consisting of two multi-wire drift chambers and plastic scintillation detectors allowed the reconstruction of the scattering angle at the target via ray-tracing techniques [9].

Typical spectra for the ${ }^{11} \mathrm{~B}\left(\alpha, \alpha^{\prime}\right)$ and ${ }^{13} \mathrm{C}\left(\alpha, \alpha^{\prime}\right)$ reactions are shown in Fig. 1. Energy resolutions of the excitation energy spectra were $250 \mathrm{keV}$ and $180 \mathrm{keV}$ for ${ }^{11} \mathrm{~B}$ and ${ }^{13} \mathrm{C}$ at full width at half maximum, respectively. The energy resolution for ${ }^{13} \mathrm{C}$ was dominated by the energy spread of the cyclotron beam, whereas that for ${ }^{11} \mathrm{~B}$ was deteriorated by the energy straggling in the thick ${ }^{11} \mathrm{~B}$ target.
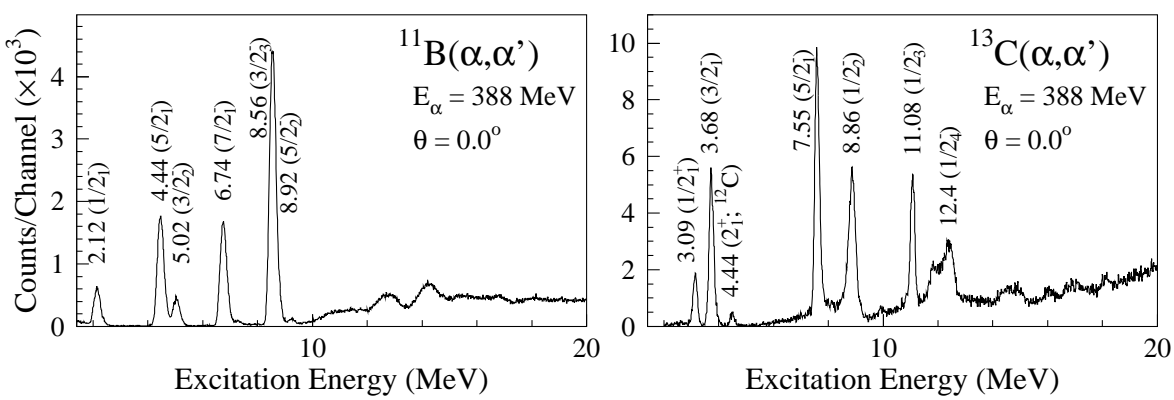

Fig. 1: Excitation energy spectra for the ${ }^{11} \mathrm{~B}\left(\alpha, \alpha^{\prime}\right)$ (left) and ${ }^{13} \mathrm{C}\left(\alpha, \alpha^{\prime}\right)$ (right) reactions measured at $0^{\circ}$.

\section{Result and discussion}

The measured cross sections for the ${ }^{13} \mathrm{C}\left(\alpha, \alpha^{\prime}\right)$ and ${ }^{11} \mathrm{~B}\left(\alpha, \alpha^{\prime}\right)$ reactions exciting the several low-lying states are compared with the theoretical predictions by the distorted-wave Born approximation (DWBA) calculation in Figs. 2. The transition potentials in the DWBA calculation were obtained by folding the macroscopic transition densities [10] with the phenomenological $\alpha N$ interaction $V_{\alpha N}(r)$ given by:

$$
V_{\alpha N}(r)=-V \exp \left(-r^{2} / \alpha_{V}\right)-i W \exp \left(-r^{2} / \alpha_{W}\right) .
$$

The interaction strengths and range parameters of $V=16.9 \mathrm{MeV}, W=11.7 \mathrm{MeV}$, and $\alpha_{V}=\alpha_{W}=$ $4.38 \mathrm{fm}^{2}$ were determined to reproduce the cross section for the elastic scattering from ${ }^{12} \mathrm{C}$.
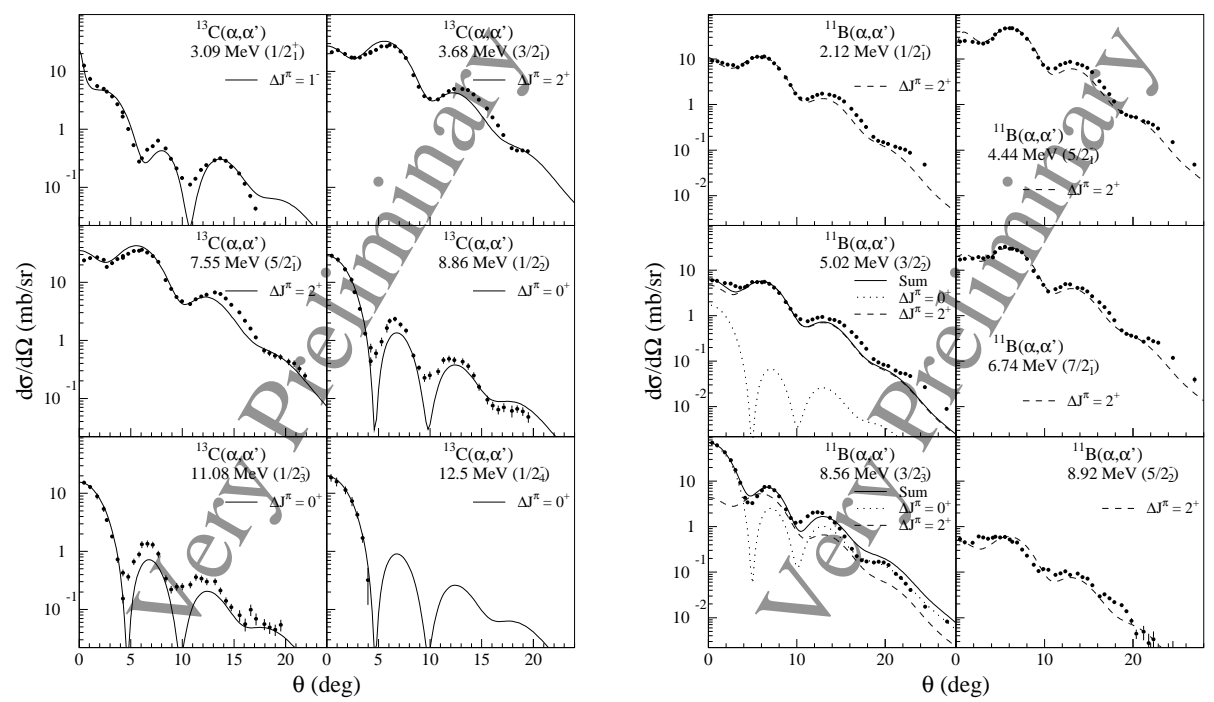

Fig. 2: Preliminary results of the measured cross sections for the several low-lying states in ${ }^{13} \mathrm{C}$ (left) and ${ }^{11} \mathrm{~B}$ (right) compared with the DWBA calculation. 
The cross sections for the $1 / 2^{-}$and $1 / 2^{+}$states in ${ }^{13} \mathrm{C}$ peak at $0^{\circ}$, and rapidly decrease with the increasing scattering angle. The allowed transferred spin and parity are uniquely defined in the ${ }^{13} \mathrm{C}\left(\alpha, \alpha^{\prime}\right)$ reaction since the spin-parity of the ground state of ${ }^{13} \mathrm{C}$ is $1 / 2^{-}$and only the natural-parity transitions are allowed in the inelastic $\alpha$ scattering. Therefore, it is naturally noted that the $1 / 2^{-}$states are excited by the monopole transitions whereas the enhancement of the $1 / 2^{+}$state near $0^{\circ}$ is due to the dipole Coulomb excitation.

On the other hand, several multipole transitions are allowed in the ${ }^{11} \mathrm{~B}\left(\alpha, \alpha^{\prime}\right)$ reaction. The ${ }^{11} \mathrm{~B}\left(\alpha, \alpha^{\prime}\right)$ cross sections were analyzed by summing up the calculated cross sections for the allowed multipole transitions with $\Delta J \leq 2$.

The deformation lengths in the macroscopic transition densities were determined to reproduce the measured cross sections for the ${ }^{13} \mathrm{C}\left(\alpha, \alpha^{\prime}\right)$ and ${ }^{11} \mathrm{~B}\left(\alpha, \alpha^{\prime}\right)$ reactions, and the isoscalar monopole excitation strengths $B(E 0 ; I S)$ for the $1 / 2^{-}$states in ${ }^{13} \mathrm{C}$ and the $3 / 2^{-}$states in ${ }^{11} \mathrm{~B}$ were obtained from the deformation lengths as listed in Table 1.

Table 1: Preliminary results of the isoscalar monopole excitation strengths for the $1 / 2^{-}$states in ${ }^{13} \mathrm{C}$ and the $3 / 2^{-}$ states in ${ }^{11} \mathrm{~B}$.

\begin{tabular}{cccccc}
\hline \hline & \multicolumn{2}{c}{${ }^{13} \mathrm{C}$} & \multicolumn{3}{c}{${ }^{11} \mathrm{~B}$} \\
$J^{\pi}$ & $\begin{array}{c}E_{x} \\
(\mathrm{MeV})\end{array}$ & $\begin{array}{c}B(E 0 ; I S) \\
\left(\mathrm{fm}^{4}\right)\end{array}$ & $J^{\pi}$ & $\begin{array}{c}E_{x} \\
(\mathrm{MeV})\end{array}$ & $\begin{array}{c}B(E 0 ; I S) \\
\left(\mathrm{fm}^{4}\right)\end{array}$ \\
\hline $1 / 2_{2}^{-}$ & 8.86 & $41 \pm 6$ & $3 / 2_{2}^{-}$ & 5.02 & $5 \pm 3$ \\
$1 / 2_{3}^{-}$ & 11.08 & $23 \pm 3$ & $3 / 2_{3}^{-}$ & 8.56 & $88 \pm 15$ \\
$1 / 2_{4}^{-}$ & 12.5 & $29 \pm 4$ & & & \\
\hline \hline
\end{tabular}

The three $1 / 2^{-}$states in ${ }^{13} \mathrm{C}$ and the $3 / 2_{3}^{-}$state in ${ }^{11} \mathrm{~B}$ are strongly excited by the isoscalar monopole transitions, but those large monopole strengths cannot be explained by the shell-model calculation at all. This fact indicates that the structure of these states is quite different from the shell-model picture where each nucleon behaves like an independent particle in the mean-field potential. The nonshell-model-like structure of those states is possibly due to the $\alpha$-cluster correlation. It is generally difficult to treat the clustering phenomena in the truncated shell-model space since the theoretical description of the clustering phenomena under the shell-model framework requires a huge number of single-particle bases. Actually, the antisymmetrized molecular-dynamics calculation shows the large monopole strength for the $3 / 2_{3}^{-}$state in ${ }^{11} \mathrm{~B}$ is well described by a spatially well-developed $2 \alpha+t$ cluster wave function [11].

Recently, it is theoretically pointed out that a sizable monopole strength could be a signature of the $\alpha$ cluster states [7]. Thus, it should be noted that the three $1 / 2^{-}$states in ${ }^{13} \mathrm{C}$ are candidates for the $\alpha$ cluster states with a $3 \alpha+n$ molecular configuration. For further clarification, a quantitative comparison between the present result and the cluster-model calculations is desired. The results will be reported elsewhere soon.

\section{Summary}

The inelastic $\alpha$ scattering at $E_{\alpha}=388 \mathrm{MeV}$ was measured to examine the $\alpha$ cluster structures in ${ }^{11} \mathrm{~B}$ and ${ }^{13} \mathrm{C}$. The measured cross sections for the low-lying states were compared with the DWBA calculation, and the isoscalar monopole strengths were determined. It was found that the $1 / 2_{2}^{-}, 1 / 2_{3}^{-}$, and $1 / 2_{4}^{-}$ states in ${ }^{13} \mathrm{C}$ are candidates for the $\alpha$ cluster states with a $3 \alpha+n$ molecular configuration. For further clarification, a quantitative comparison between the present result and the cluster-model calculations is desired. The results will be reported elsewhere soon. 


\section{Acknowledgements}

The author would like to thank the members of RCNP-E253 and E308 for their collaboration. The author acknowledges the effort of the RCNP cyclotron crew for providing the stable and clean beam. This work was supported in part by the Grant-in-Aid for Scientific Research No. 17740132 and for the Global COE Program "The Next Generation of Physics, Spun from Universality and Emergence" from the Ministry of Education, Culture, Sports, Science and Technology (MEXT) of Japan.

\section{References}

[1] K. Ikeda, N. Takigawa, and H. Horiuchi, Prog. Theor. Phys. Suppl. Extra Number (1968) 464.

[2] H. Morinaga, Phys. Rev. 68 (1956) 29.

[3] M. Milin and W. von Oertzen, Euro. Phys. J. A 14 (2002) 295.

[4] T. Kawabata, H. Akimune, H. Fujita, Y. Fujita, M. Fujiwara, K. Hara, K. Hatanaka, M. Itoh, Y. Kanada-En'yo, S. Kishi, K. Nakanishi, H. Sakaguchi, Y. Shimbara, A. Tamii, S. Terashima, M. Uchida, T. Wakasa, Y. Yasuda, H. P. Yoshida, and M. Yosoi, Phys. Lett. B 646 (2007) 6.

[5] S. Cohen and D. Kurath, Nucl. Phys. 73 (1965) 1.

[6] T. Kawabata, H. Akimune, H. Fujimura, H. Fujita, Y. Fujita, M. Fujiwara, K. Hara, K. Y. Hara, K. Hatanaka, T. Ishikawa, M. Itoh, J. Kamiya, S. Kishi, M. Nakamura, K. Nakanishi, T. Noro, H. Sakaguchi, Y. Shimbara,

H. Takeda, A. Tamii, S. Terashima, H. Toyokawa, M. Uchida, H. Ueno, T. Wakasa, Y. Yasuda, H. P. Yoshida, and M. Yosoi, Phys. Rev. C 70 (2004) 034318.

[7] T. Yamada, H. Horiuchi, K. Ikeda, Y. Funaki, and A. Tohsaki, Preprint nucl-th/0703045.

[8] M. Fujiwara, H. Akimune, I. Daito, H. Fujimura, Y. Fujita, K. Hatanaka, H. Ikegami, I. Katayama, K. Nagayama, N. Matsuoka, S. Morinobu, T. Noro, M. Yoshimura, H. Sakaguchi, Y. Sakemi, A. Tamii, and M. Yosoi, Nucl. Inst. \& Meth. in Phys. Res. A 422 (1999) 484.

[9] T. Kawabata, T. Ishikawa, M. Itoh, M. Nakamura, H. Sakaguchi, H. Takeda, T. Taki, M. Uchida, Y. Yasuda, M. Yosoi, H. Akimune, K. Yamasaki, G. P. A. Berg, H. Fujimura, K. Hara, K. Hatanaka, J. Kamiya, T. Noro, E. Obayashi, T. Wakasa, H. P. Yoshida, B. A. Brown, H. Fujita, Y. Fujita, Y. Shimbara, H. Ueno, M. Fujiwara, K. Hosono, A. Tamii, and H. Toyokawa, Phys. Rev. C 65 (2002) 064316.

[10] M. Harakeh and A. van der Woude, Giant Resonances (New York: Oxford University Press, 2001).

[11] Y. Kanada-En'yo, Phys. Rev. C 75 (2007) 024302. 